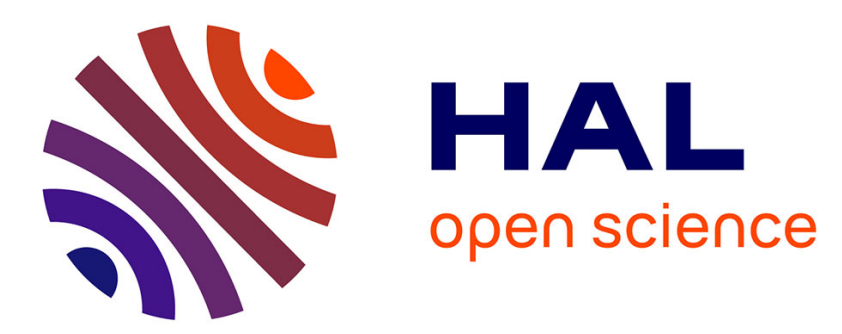

\title{
Distributed Monitoring of Peer to Peer Systems
}

Serge Abiteboul, Bogdan Marinoiu

\section{- To cite this version:}

Serge Abiteboul, Bogdan Marinoiu. Distributed Monitoring of Peer to Peer Systems. ACM WIDM 2007, ACM, Nov 2007, Lisbon, Portugal. inria-00259054

\section{HAL Id: inria-00259054 https://hal.inria.fr/inria-00259054}

Submitted on 26 Feb 2008

HAL is a multi-disciplinary open access archive for the deposit and dissemination of scientific research documents, whether they are published or not. The documents may come from teaching and research institutions in France or abroad, or from public or private research centers.
L'archive ouverte pluridisciplinaire HAL, est destinée au dépôt et à la diffusion de documents scientifiques de niveau recherche, publiés ou non, émanant des établissements d'enseignement et de recherche français ou étrangers, des laboratoires publics ou privés. 


\title{
Distributed Monitoring of Peer to Peer Systems
}

\author{
Serge Abiteboul and Bogdan Marinoiu \\ INRIA Saclay and University Paris Sud \\ 4 , rue Jacques Monod \\ 91893 Orsay CEDEX, France \\ firstname.lastname@inria.fr
}

\begin{abstract}
In this paper, we are concerned with the distributed monitoring of P2P systems. We introduce the P2P Monitor system and a new declarative language, namely P2PML, for specifying monitoring tasks. A P2PML subscription is compiled into a distributed algebraic plan which is described using algebra over XML streams. The operators of this algebra are first alerters in charge of detecting specific events and acting as stream sources. Other operators process the streams or publish them.

We introduce a filter for streams of XML documents that scales by processing first simple conditions and then, if still needed, evaluating complex queries. We also show how particular tasks can be supported by identifying subtasks that are already provided by existing streams.
\end{abstract}

\section{Categories and Subject Descriptors}

H.2 [Database management]: Distributed databases, Query processing

\section{General Terms}

Algorithms, Design, Languages, Performance

\section{Keywords}

distributed data management, stream processing, peer to peer systems, databases, Web services, active documents

\section{INTRODUCTION}

Peer to Peer systems have become popular over the last decade mainly because they provide support for community content sharing and for loosely coupled distributed applications. Their use is still hindered by the difficulty to observe such highly dynamic systems, and to gather information on their functioning. Observation turns out to be essential in many contexts, e.g., error management, statistics gathering, workflow control, Web surveillance. This is the topic of the present paper where we propose a generic monitoring system

Permission to make digital or hard copies of all or part of this work for personal or classroom use is granted without fee provided that copies are not made or distributed for profit or commercial advantage and that copies bear this notice and the full citation on the first page. To copy otherwise, to republish, to post on servers or to redistribute to lists, requires prior specific permission and/or a fee.

WIDM'07, November 9, 2007, Lisbon, Portugal.

Copyright 2007 ACM 978-1-59593-829-9/07/0011 ...\$5.00. for P2P systems. A main contribution is that subscriptions, specified in a declarative language, are compiled into distributed algebraic plans over XML streams. We present a new algorithm for efficient filtering and introduce a novel $\mathrm{P} 2 \mathrm{P}$ technology for re-using already existing streams.

We introduce a system, called P2P Monitor (P2PM for short) for monitoring $\mathrm{P} 2 \mathrm{P}$ systems. $\mathrm{P} 2 \mathrm{PM}$ is itself a $\mathrm{P} 2 \mathrm{P}$ system. So, we have two P2P systems that coexist, the monitored one (possibly several monitored systems) and the monitoring one (namely P2PM). The same machine may participate in both kinds of $\mathrm{P} 2 \mathrm{P}$ networks. Each peer in the monitored P2P system can observe some activities (e.g. data changes or communications) happening locally and thus become a primary source of monitoring information. We represent such information as a stream of XML data. Such data may be transmitted between peers through channels (e.g., using point to point or broadcasting). The peers in P2PM perform operations on data streams to produce new streams or publish resulting streams.

A main characteristic of the system is the use of a declarative Subscription Language, P2PML, short for Peer-to-Peer Monitor Language. A monitoring task is specified to the system in this language. It is then compiled into an algebraic monitoring plan involving both the monitored system and P2PM. Thus, our work is founded on an algebra over data streams (i.e., a library of services) derived from the ActiveXML algebra framework for distributed data management [4]. The algebraic operators include Alerters - 0-ary operators, that are situated on the premises of the monitored peers, detect specific local events and produce data streams, stream processors - for filtering or applying more complex operations on streams, and publishers - for exposing streams to other peers of the system (in channels), or to the human users in e-mails, RSS feeds or Web pages.

The most important stream processor is the Filter, which can perform efficiently a large number of filtering queries over a stream with intense traffic. An important aspect, from a performance viewpoint, is that it checks separately simple test conditions, evaluated on the fly, and more complex ones that require the use of an XML query processor.

An important issue for scaling with many subscriptions and peers is the placement of operators such as filters close to the data they work on when possible, to save on data transfers. As we will see, ActiveXML is also used for reducing the amount of data that is transferred by providing information intentionally when possible to avoid useless transfers and replication. With respect to replication, P2PM also includes the means to reduce the load on the system by re-using ex- 
isting data streams. When a new monitoring subscription arrives, the system searches for existing streams that could help support (portions of) the new task. This (monitoring) service discovery is implemented on top of a P2P content management system, namely KadoP [3].

Motivations. A wide range of applications can benefit from such a monitoring of $\mathrm{P} 2 \mathrm{P}$ systems. We will mention briefly some that particularly motivated the present work.

P2PM can be used to observe Web services activity in a Web community. Towards this goal, we implemented an alerter to monitor SOAP messages. This could serve, for instance, to follow the concurrent execution of large number of workflow instances in telecom services (e.g., BPEL workflows [6]) to detect malfunctions, gather statistics, understand usage patterns, support billing, etc.

An application we considered for testing is the surveillance of the content published by Web servers (e.g., for a community portal). In particular, we developed an alerter to monitor changes in RSS feeds.

A main motivation of our work is the monitoring of the Edos content sharing network [9]. Edos is a P2P distribution system that is developed in cooperation with the Mandriva company (originally MandrakeSoftware). In Edos, the data consists of the Mandriva Linux distribution, i.e., about 10000 software packages and the associated metadata. The metadata for one distribution is more than 100 megabytes of XML data. The monitoring is primarily used to gather statistics about the peers (e.g., number, efficiency, reliability) and the usage of the system (e.g., query rate).

The paper is organized as follows. Section 2 introduces the subscription language. Section 3 defines the notions of streams and channels and presents the architecture of P2PM as well as the Stream Algebra. Section 4 focuses on the module implementing efficiently stream filtering. Section 5 shows techniques allowing stream reuse. In Section 6, we compare $P 2 P M$ to other systems and in Section 7, we conclude.

\section{P2P MONITORING LANGUAGE}

In this section, we briefly describe the subscription language and thereby the functionalities of the system.

The monitoring language P2PML allows specifying in declarative statements, called (monitoring) subscriptions, complex events a user is interested in, as well as how the user should be notified of detected events. The system is globally in charge of performing the corresponding (monitoring) task, and in particular, of assigning the operators.

For instance, consider the subscription involving three peers in Figure 1. The monitor office of meteo.com wants to detect when the meteo service it provides to some peers, a.com and b.com, is too slow (takes more than 10s).

As one can notice, the syntax is inspired by the XQuery's FLWR [18]. Statements in the language use five types of clauses that are discussed next.

The FOR clause specifies the information sources. Three sources are used in the example: two alerters of outgoing calls (outCOM), one on peer $a$, one on $b$, and an alerter of incoming calls (inCOM) at meteo.com. Note that the same "call" is an out-call for a.com and an in-call for meteo.com. The clause defines XML variables: $\$ c 1$ for events detected by the two clients and $\$ c 2$ for events detected at the server.

The functions in the FOR clause define the nature of the alerters that are used. In the example, the outCOM and in $C O M$ alerters monitor the communications in SOAP RPC

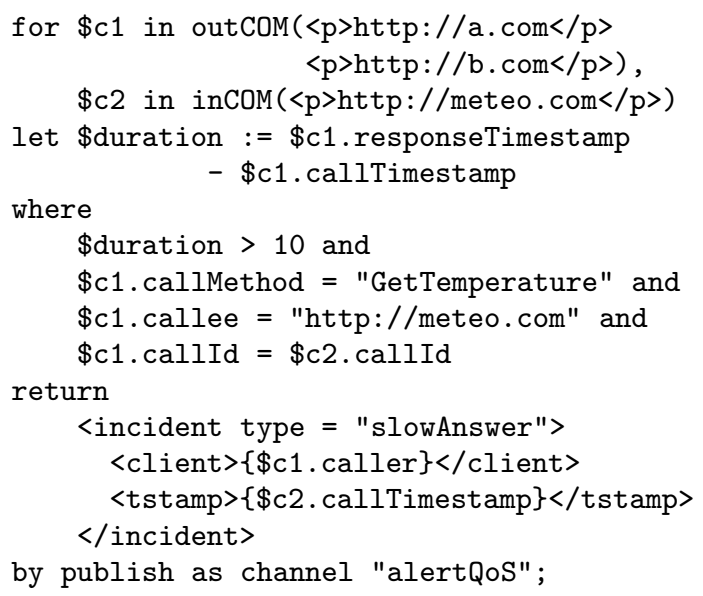

Figure 1: A monitoring subscription

calls. Such communications consist of a pair of a Call and a Response. An alerter produces a stream of XML trees. An element of such stream is called a stream item. In a stream item, we distinguish two parts:

1. the attributes of the root that typically gather some generic information. For a Web service alerter, these will include call identifier, server identifier or the time of the call. Selection conditions on these attributes are very common in subscriptions.

2. the sub-elements of the root that possibly have some more complex structure. In the case of the Web service alerter, the entire SOAP message or an error message may be included in the alert.

The filter will process selections over these two kinds of data in the alert differently for performance reasons.

The LET clause enables the user to define more variables based on the already defined ones, e.g. Sduration.

The WHERE clause imposes some Boolean conditions on the variables. (For the moment, the system supports only conjunctions of conditions.) The conditions are equality or inequality conditions on the atomic variables (integer or strings) or on some atomic values that can be extracted from variables using XPath queries. An example of a XPath query is:

\section{\$c1/alert [@callMethod = "GetTemperature"]}

Since such conditions on the root attributes of alerts are very common, we use a dot notation as syntactic sugaring. For instance, the previous condition can be expressed as: \$c1.callMethod = "GetTemperature". Conditions on the root attributes are called simple conditions. The Where clause in the example contains four simple conditions.

The RETURN clause specifies the output stream. When conditions are matched by the values over the input streams, an output XML tree is obtained in the output stream. This output is defined as XML data with possibly curly bracketsguarded expressions that are to be evaluated at runtime.

Finally, the $B Y$ clause determines how the user gets notified. Publication in a channel (the most interesting case), illustrated by the example, consists in publishing a stream that clients (other peers) can subscribe to or other subscriptions (issued by human users) can refer to. This will be detailed in Section 3.

As previously mentioned, the main concept of the system is the stream. A subscription produces a stream. Furthermore, the syntax declaration of a FOR clause is: 


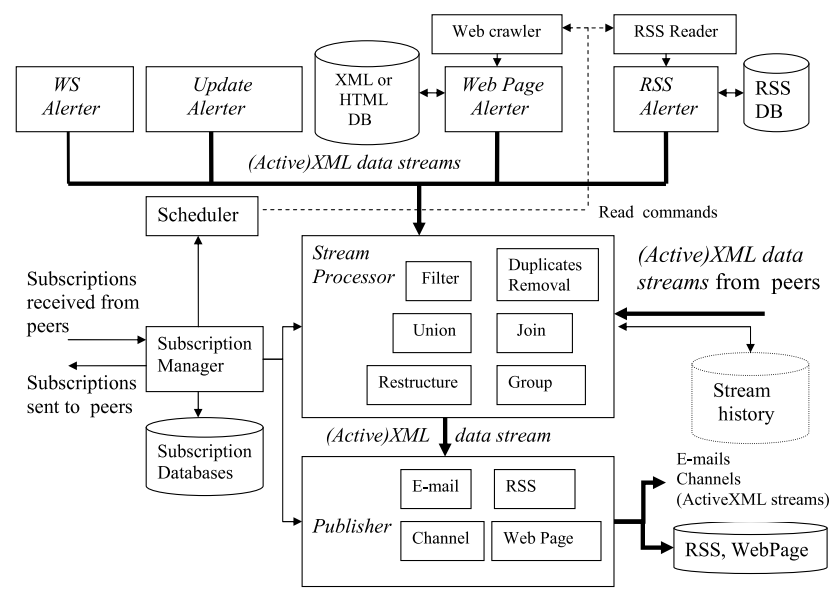

Figure 2: The architecture of a peer in P2PM

<fclause> :- FOR <var> in <stream>

(, <var in <stream>) $*$

So, in particular we can nest subscriptions:

for $\$ \mathrm{x}$ in ( for $\$ y$ in ... . . .

Also, functions such as inCOM take a stream as input and produce a stream as output. The input stream does not have to be fixed. So for instance, one can define:

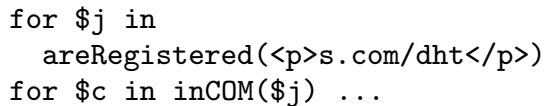

For this example we suppose that our system has the support of a DHT and that the DHT exports a stream of events, corresponding to peers joining or leaving:

$\langle p-$ join $>$ a.com $</ p-j o i n>\%$ a joins

$<\mathrm{p}-$ leave $>$ a. com $</ \mathrm{p}-$ leave $>\%$ a leaves

In the latter case, inCOM removes peers from the collection of monitored peers.

One can also request duplicate-free results by preceeding the content of the RETURN clause by the distinct attribute as in: return distinct $\langle a\rangle\{\$ y\}</ a>$.

The syntax of P2PML has the flavor of that of XQuery. Clearly, it is very different because its role is not to query $\mathrm{XML}$ documents but to monitor $\mathrm{P} 2 \mathrm{P}$ systems; in particular the BY clause has no analogue in XQuery. XQuery has an influence because the streams contain XML data.

\section{P2P MONITOR}

We first present the architecture of P2PM. Then we focus on two key aspects: (i) the ActiveXML algebra that is used in the system and (ii) the generation of monitoring plans.

\subsection{Architecture}

The functional architecture of a peer in the P2PM is shown in Figure 2. Between them and with other peers, the modules mostly exchange streams of ActiveXML trees. The minimum required to be a P2PM peer is to run a Subscription Manager. A peer may also host some alerters, stream processors and some publishers. These are discussed next.

Subscription manager. When a user requests a monitoring task in P2PML, she forwards the subscription to a peer which becomes Subscription Manager for this subscription. A peer keeps the information about all subscriptions under his responsibility in a database named Subscription Database.
The Subscription Manager is in charge of translating the subscription into a monitoring plan, optimizing this plan, and then deploying the optimized plan (See Section 3.4). A peer can also delegate a part of the monitoring task, by expressing demands in the same P2PML language for monitoring subtasks to other peers.

Alerters. Each alerter (a 0-ary operator producing a stream) is specialized in detecting particular events in some systems that are external to P2PM, as described next. An $W S$ Alerter intercepts inbound-outbound Web service calls and produces alerts including SOAP envelopes expanded with annotations such as timestamps and the identifiers (DNS/IP) for caller/called entities. They are implemented as Axis handlers. An ActiveXML alerter detects updates to the ActiveXML peer's repository. A WebPage Alerter detects changes in XML/XHTML pages by comparing their snapshots. The alert may provide (if desired) the delta between two pages. (This alerter uses an auxiliary Web crawler for the surveillance of collections of Web pages). RSS Feed Alerter detects changes in an RSS feed by comparing snapshots also. With RSS, the alerts have more semantics than with arbitrary XML: e.g., add, remove and modify entry.

Stream processors. Some of the stream processors are stateless, i.e., their behavior does not depend on the history of their input streams, e.g., Filter $(\sigma)$, Restructure (П), Union $(\cup)$. Others are stateful, e.g., Duplicate-removal, Join $(\bowtie)$ or Group. We next briefly discuss the main processors we support. The Filter processor whose performance is critical for the usability of the system, is discussed in Section 4.

Join takes two streams as input and generates an output stream. Join can be parameterized by a join predicate. For instance, in Section 2, we need to "join" the alerts of the two streams using the equality of the callIds. Such a join is typically very used in monitoring systems to follow a task across different peers. For each new tree $t$ in one of the input streams, the history of the other stream is searched for a tree $t^{\prime}$ so that $\left(t, t^{\prime}\right)$ matches the join predicate. An index over that history is used to speed up the search. The result of Join includes information about the matching pair of trees. Duplicate-removal detects similar trees based on a duplicate criteria. Union takes several streams as inputs and merges them into a single stream.

Restructure takes as input one stream. A template defines the restructuring that has to be done at runtime based on the input. In the simplest case, the template specifies a projection of an input tree. For the input tree that passed the Where test (or for a tuple of trees, e.g., in case of joins), the template is used to construct the output tree.

Publisher. Publisher is an operator in charge of publishing streams generated by stream processors, under different forms: by emails, in XML files (ordinary XML documents, XHTML Web pages or RSS feeds) or as channels. The chan$n e l$ is the basis of our Pub/Sub mechanism. An entity interested in some stream, has to subscribe to a channel publishing it. If a peer $P_{1}$ is interested in the output stream of a service evaluating at $P_{2}$, it asks $P_{2}$ to publish its results on a channel $\# x$. $P_{2}$ then subscribes to this channel $\# x @ P_{1}$.

Implementation. P2PM is implemented in Java, on top of ActiveXML Peer. It is a Web application using Axis libraries to handle SOAP Web services and the Jakarta Tomcat servlet engine. JavaCC libraries are used to build a 
parser for P2PML. Code from the YFilter [8] project was modified and used to implement an adaptable automatabased query processor for XML document streams.

\subsection{Streams, channels, services}

The language ActiveXML [5] has been proposed to support distributed query evaluation and optimization. The Active XML algebra [4] is an algebra over (Active) XML streams. We show here how it can serve as the basis of a $\mathrm{P} 2 \mathrm{P}$ monitoring system. Indeed, $P 2 P M$ is based on the exchange of ActiveXML streams and it uses the ActiveXML algebra. We briefly recall some definitions of ActiveXML and sketch the ActiveXML algebra.

An ActiveXML document is an XML document where some of the elements ( $s c$ elements), denote calls to Web services. The evaluation of such a call results in performing the call and enriching the document with its result (e.g., by appending the result at the place of the call). All the information needed for performing the call (e.g., for accessing the service, deciding when to perform the call or what to do with the result) is provided inside the $s c$ element.

An XML (respectively, ActiveXML) stream is a possibly infinite sequence of XML (respectively, ActiveXML) trees. A particular symbol eos may be considered to denote the termination of the stream. Typically, a stream is sent from one peer to a set of peers using the concept of channel.

A channel is defined by a tuple (peerID, streamID, subscribers), where peerID is the peer that published this particular stream as a channel and subscribers is the set of peers interested in it. Note that subscribing to a monitoring task is different from subscribing to a channel. A monitoring task subscription is defining a complex distributed interaction between peers. Subscribing to a channel means expressing the will to receive the data published by the channel.

A subscription to a channel can be seen as a call to a service, where the result of the service call is a stream of ActiveXML trees. In ActiveXML terminology, this is a continuous service. The trees in the stream are received one after another in an asynchronous manner. Note that a noncontinuous service is a particular case: the service returns an ActiveXML tree followed by an eos.

We adopt the following ActiveXML notation. A document $d$ or a service $s$ at peer $p$, are denoted respectively $d @ p, s @ p$. Some services, called generic, can be offered by many peers. In particular, query services can be offered by any peer with an XML query processor. Such a service is denoted $s @ a n y$, e.g., $\sigma_{/ / a / / b} @ a n y$. (In the following, a service $s$ with no peer location is assumed to be $s @ a n y$.$) For deployment,$ the generic services will be replaced by concrete ones.

\subsection{The Stream Algebra}

We next briefly present the stream algebra. Details may be found in [4]. As in [4], we consider the following alphabets: $\mathcal{D}$ of document names, $\mathcal{S}$ of service names, $\mathcal{P}$ of peer identifiers, $\mathcal{N}$ of node identifiers, $\mathcal{L}$ of label identifiers and $\mathcal{V}$ of variables. Data variables are denoted as $\$ x, \$ y \ldots$ and node variables as $\sharp x, \sharp y \ldots$. The set $\mathcal{S}$ contains particular services: send, receive and eval. ActiveXML expressions are used to model distributed evaluations. For $l \in \mathcal{L}, p$ and $p^{\prime}$ peers, $d @ p$ a document at $p, s @ p$ a service of arity $k, n @ p$ a node in some document at peer $p$, and the algebraic expressions $e_{1}, e_{2} \ldots e_{k}$, the following are also algebraic expressions:

$l\left\langle e_{1}, \ldots, e_{k}\right\rangle, s @ p\left(e_{1}, \ldots, e_{k}\right), d @ p, e v a l @ p\left(e_{1}\right)$, send@p $\left(n @ p^{\prime}, e_{1}\right)$, receive@p () .

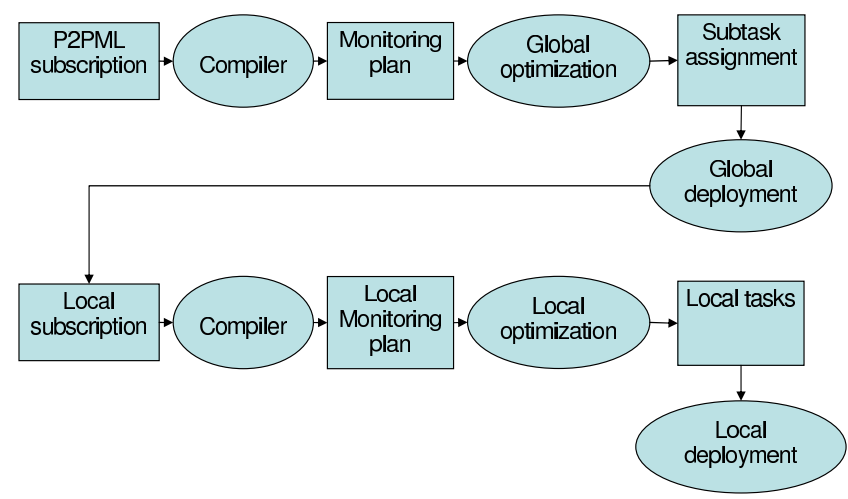

Figure 3: Subscription Processing Chains

An executing service $s @ p$ is noted os@ $p$. A service that finished executing is noted $\bullet s @ p$. eval@ $p\left(s @ p^{\prime}(\ldots)\right)$ means $p$ asks for the execution of $s @ p^{\prime}(\ldots)$ on peer $p^{\prime}$.

The semantics of algebraic expressions are defined using rewriting rules. To illustrate them, we present only the two rules for service invocation.

1. Local service invocation

$$
\begin{array}{r}
x_{0} @ p: \text { eval@p }\left(s @ p\left(\ldots, t_{i}, \ldots\right)\right) \rightarrow \\
x_{0} @ p: o s @ p\left(\ldots, \text { eval@ } p\left(t_{i}\right), \ldots\right)
\end{array}
$$

2. External service invocation

$$
\begin{array}{r}
\sharp x @ p\left\langle e v a l @ p\left(s @ p^{\prime}(\ldots)\right)\right\rangle \rightarrow \\
\sharp x @ p\langle\text { oreceive@p }()\rangle \&
\end{array}
$$$$
(n e w) @ p^{\prime}: e v a l @ p^{\prime}\left(\operatorname{send} @ p^{\prime}\left(\sharp x @ p,\left(s @ p^{\prime}(\ldots)\right)\right)\right)
$$

In the second rule, Peer $p$ asks $p^{\prime}$ to evaluate service $s @ p^{\prime}$ (e.g., a local database call at $p^{\prime}$ ) and to send its (stream of) result(s) under the node $\sharp x @ p$. Note the fact that $p$ is executing receive() to accept data from $p^{\prime}$ and to place it at the right spot. The separator \& between actions means that these are done concurrently (here, at peers $p$ and $p^{\prime}$ ).

We also present an important rule for query optimization that illustrates the tight interaction with the local optimizer: If $q \equiv q^{\prime}\left(q_{1}, \ldots, q_{n}\right)$, then

$$
\begin{array}{r}
\text { eval@p }(q @ a n y) \leftrightarrow \\
\text { eval@p }\left(q^{\prime} @ a n y\left(q_{1} @ a n y, \ldots, q_{n} @ a n y\right)\right)
\end{array}
$$

Let us consider again the example of Section 2 and denote by out@a.com and out@b.com the two alerters over outgoing calls, andin@meteo.com the alerter on incoming calls. Let $p$ be the peer that processes the subscription. Then the subscription is first compiled into the plan:

$$
\begin{gathered}
\text { eval@p } p\left(\text { publisher } \left(\Pi_{T}(\right.\right. \\
\bowtie_{P}\left(\cup \left(\sigma_{F}(\text { out } @ \text { a.com }),\right.\right. \\
\left.\left.\sigma_{F}(\text { out } @ \text { b.com })\right)\right), \\
\left.\left.\left.\sigma_{F^{\prime}}(\text { in } @ \text { meteo.com })\right)\right)\right)
\end{gathered}
$$

where $T$ is the restructuring template, $P$ the join predicate, $F, F^{\prime}$ some filtering over the out and in-calls, respectively. Observe that in the above expression, some services are still generic (i.e., non concrete). Observe that the selections were pushed as much as possible to the proximity of the sources to save on communications and that operators have not yet been "placed" with the exception of the alerters.

\subsection{Monitoring plan generation}

As already mentioned, the subscription manager is in charge of compiling a subscription into a monitoring plan that will then be optimized. The monitoring plans are expressed in the algebra using the discussed operators, in particular, 


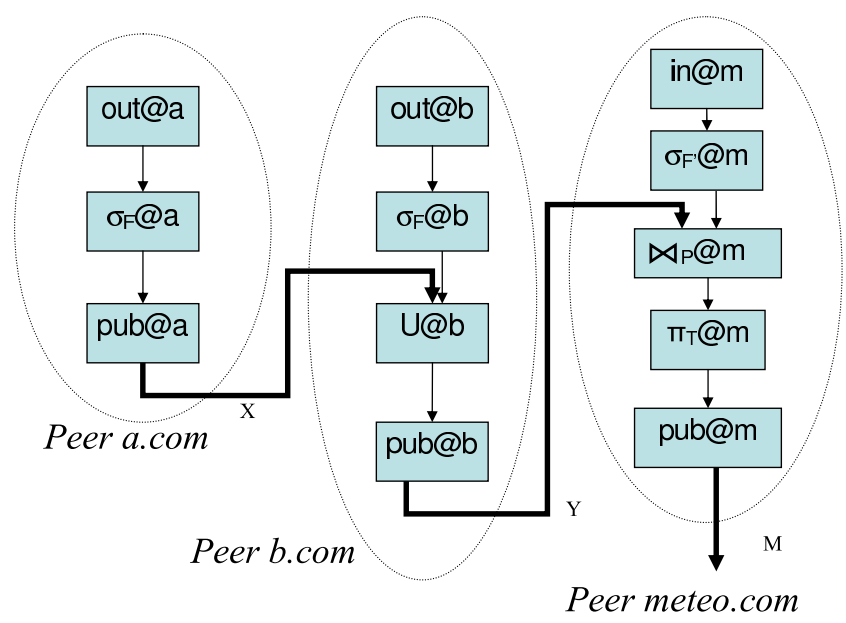

Figure 4: One possible plan for the monitoring task alerters, stream processors and publishers. Figure 3 presents the steps transforming a subscription into a running monitoring task. The processing phases are represented by ovals and the input/output data by rectangles. Observe that at the end of the top processing chain, the work is distributed between the peers in the system. Some of the work may be requested locally (observe the bottom processing chain).

In a first step, the subscription manager computes an optimized plan for the given subscription. The optimization is performed using algebraic rewrite rules and heuristics. In a second step, it searches for resources in the system that cover at least parts of the task plan. This is considered in Section 5. Finally, for the new tasks, the subscription manager assigns them to peers, trying to balance the load.

To illustrate the first step, let us consider again the example. Imagine that no existing stream was found that could serve (part of) the subscription. Suppose that the query optimizer selects the plan (using the ActiveXML syntax):

$$
\begin{aligned}
& \text { eval@p(publisher@p }\left(\Pi_{T} @ \text { meteo.com }(\right. \\
& \bowtie_{P} @ \text { meteo.com }\left(\cup @ b . c o m\left(\sigma_{F} @ a . c o m(\text { out } @ a . c o m),\right.\right. \\
& \left.\left.\sigma_{F^{\prime}} @ \text { meteo.com }(\text { in } @ \text { meteo.com })\right)\right) \text { ) }
\end{aligned}
$$

By rewriting, this yields:

$\%$ at $\mathrm{p}$

$$
\text { opublisher@p( } \sharp M @ p: \text { oreceive }())
$$

$\& \%$ at meteo.com

osend@meteo.com( $\sharp M @ p, \circ \Pi_{T} @ m e t e o . c o m($ $\circ \bowtie_{P} @$ meteo.com ( $\sharp Y @$ meteo.com : oreceive (), $\circ \sigma_{F^{\prime}} @$ meteo.com $($ in@meteo.com $\left.\left.)\right)\right)$ )

$\& \%$ at b.com

osend@b.com( $\sharp Y @$ meteo.com, $\circ \cup @ b . c o m(\sharp X @ b . c o m:$ oreceive () , $\left.\circ \sigma_{F} @ b . c o m(o u t @ b . c o m)\right)$

$\& \%$ at a.com

osend@a.com( $\left.\sharp X @ b . c o m, \circ \sigma_{F} @ a . c o m(o u t @ a . c o m)\right)$

Peer a.com filters its out-calls and sends its result to b.com. Observe the use of identifiers in $\sharp X @ b$.com to denote the destination of the message, i.e., the place where the result of the filtering at a.com is expected. This is in fact supported by a publication in a channel, namely the $X$ channel published by $a$. This will allow the reuse of this stream if some other peer is interested in the same filtering. Peer b.com filters its own out-calls, merges with the data received

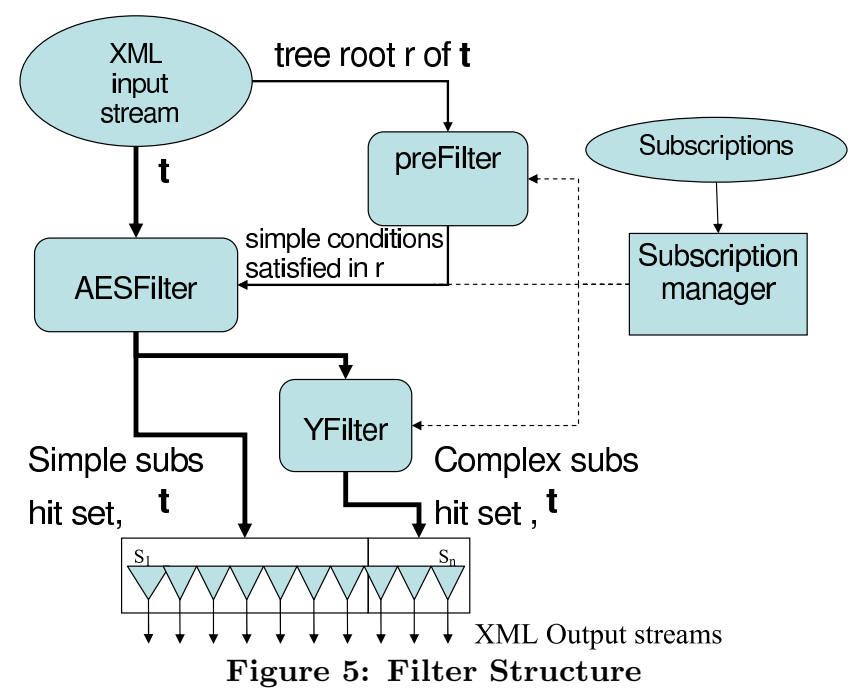

from a.com. The result of the merge is sent to meteo.com, again via a channel, this time $Y$. This last peer joins what it receives with the result of the filtering of its in-calls. Finally, meteo.com also does a transformation $\left(\Pi_{T}\right)$ to produce the results and sends it to $p$, via a last channel, namely $M$.

Observe that each expression involves only services executed at one of the peers. So, each peer can start performing its part of the global task. The same plan is represented graphically in Figure 4 for the three peers. Note that local subscriptions can also be expressed in the P2PML language.

So, for instance, Peer a.com is assigned the task:

for $\$$ e in outCOM $(\langle\mathrm{p}\rangle$ local $</ \mathrm{p}\rangle)$

let \$duration := \$e.responseTimestamp

- \$e.callTimestamp

where

\$duration > 10 and \$e.callMethod = "GetTemperature"

and $\$$ e.callee $=$ "http: $/ /$ meteo.com"

return $\$ \mathrm{e}$

by channel $\mathrm{X}$ and subscribe (b.com, \#X, X)

Observe that the result is published as a channel to which peer b.com is automatically subscribed as a first client, and $\# X$ indicates the place where this data is expected at peer b.com. Other peers may subscribe to this channel if desired.

\section{FILTER}

In this section, we describe a most important stream processor, namely Filter. As we will see, Filter is based on two basic mechanisms: the Atomic Event Set Algorithm (AES for short) [15] for matching conjunctions of simple conditions and the YFilter Algorithm [8] for matching treepatterns. The goal is to support very high volume input streams. This is of first importance for filtering potentially heavy source streams coming from alerters: e.g., telecom services, produce huge volumes of notifications to be filtered.

In Section 2, we mentioned that the attributes of the root of a stream item often contain information important for filtering. At the same time, this information is easy to access without requiring complex computation and without the need to read the entire stream item. A system can support the filtering of a very high rate of stream items on the fly if only such simple conditions are checked. This is what we do next by separating the filtering in two stages. The first step consists in checking simple conditions. The second one treating complex conditions only sees a stream of items that is typically much smaller than the stream being filtered. 


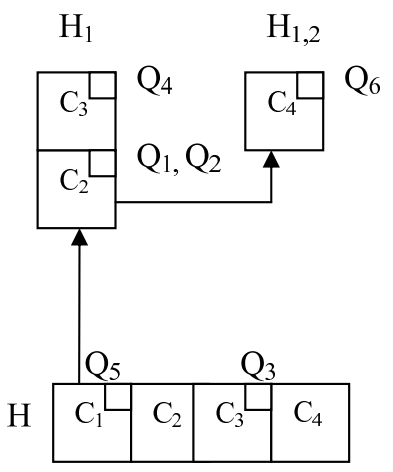

Figure 6: Atomic Event Set

More precisely, suppose we have to apply a large set $\left\{S_{i}\right\}$ of subscriptions over a stream of XML documents. A subscription $S_{i}$ is specified in Filter as a pair $\left(Q_{i}, T_{i}\right)$ where $Q_{i}$ is a conjunctive query and $T_{i}$ a report template. For each tree $t$ in the stream, Filter must find the $Q_{i}$ that matches $t$. When a matching is found, it also has to apply the template $T_{i}$ but since the main performance issue is to detect the matchings, this aspect will be ignored here. For this section, we will refer to $Q_{i}$ as subscription.

The subscriptions supported by Filter may include simple conditions, i.e., equalities or inequalities $(\neq, \leq)$ between the attributes of the root node of $t$ and constants. An example of simple condition is $\$$ c.callee $=" h t t p: / /$ meteo.com". For each $Q_{i}, Q_{i}=\wedge_{j} C_{i j}\left(\wedge Q_{i}^{\prime}\right)$ where the $C_{i j}$ are simple conditions and $Q_{i}^{\prime}$ performs the remaining complex filtering if needed. We consider here that $Q_{i}^{\prime}$ is a general tree-pattern query. If there is no $Q_{i}^{\prime}$, the subscription is said to be simple; otherwise, it is complex. Filtering is performed by three modules: preFilter, AESFilter and YFilter $_{\sigma}$.

preFilter. The preFilter module is an automaton that, for each document $t$, reads the first tag of $t$ (so, in particular, the root's attributes ). It tests the simple conditions which are organized in a hash-table with the attribute name as key and the condition as value.

AESFilter. AESFilter is a modified version of the hash tree technique of [15]. Figure 6 represents the hash tree for the subscriptions. The AES algorithm assumes that the set of simple conditions is ordered. So we assume this is the case. preFilter produces an ordered sequence of the simple conditions satisfied by $t$. AESFilter feeds that sequence in the hash tree to obtain the simple subscriptions that are satisfied by $t$ and the active complex subscriptions, i.e., those with simple conditions satisfied.

$$
\begin{aligned}
& Q_{1}=C_{1}, C_{2}, Q_{1}^{\prime} \\
& Q_{2}=C_{1}, C_{2}, Q_{2}^{\prime} \\
& Q_{3}=C_{3}, Q_{3}^{\prime} \\
& Q_{4}=C_{1}, C_{3}, Q_{4}^{\prime} \\
& Q_{5}=C_{1} \\
& Q_{6}=C_{1}, C_{2}, C_{4}, Q_{6}^{\prime}
\end{aligned}
$$

The structure used by the Atomic Event Sets algorithm is a hash-tree. The root hash-table, named $H$, has for entries simple conditions specified by the subscriptions in the system. (To simplify, we ignore subscriptions with no simple conditions.) An entry, say the entry for $C_{i_{1}}$ possibly contains a pointer to another hash table, named $H_{i_{1}}$ which contains entries for the conditions that follow condition $i_{1}$ in some subscriptions. A hash-table in this structure corresponds to a prefix in some subscription. $H_{i_{1}, i_{2}, \ldots, i_{k}}$ exists if at least one subscription has as prefix the sequence: $C_{i_{1}}, C_{i_{2}}, \ldots, C_{i_{k}}$. In the example, $H_{1,2}$ contains $C_{4}$ that follows after the sequence $C_{1}, C_{2}$ in subscription $Q_{6}$.

The structure we use to implement our AESFilter corresponds only to the simple conditions of the subscriptions. The markings correspond to the subscriptions that are still active after the processing of the simple conditions, meaning that their complex queries have to be evaluated by the YFilter $_{\sigma}$. The marked cells are the last simple conditions in at least one subscription. For instance, the condition $C_{3}$ in the hash-table $H_{1}$ is the last simple condition for $Q_{4}$. Its marking is $Q_{4}$. Details on the AES may be found in [15].

AESFilter is called with as input, the ordered list of conditions detected as valid by preFilter in the XML tree. It returns (i) the list of simple subscriptions satisfied by the tree, and (ii) the complex queries that have to be executed by the $Y$ Filter $_{\sigma}$, i.e., such that all the corresponding simple conditions are satisfied by the document. As shown in [15], this organization scales with the number of subscriptions.

YFilter $_{\sigma}$. Lastly, YFilter $\sigma$ uses the YFilter algorithm to test on $t$ the query $Q_{i}^{\prime}$ for each active subscription $Q_{i}$. Observe that we run a different filter YFilter $_{\sigma}$, depending on the complex subscriptions that passed the AESFilter test. If we suppose $t$ satisfies $C_{1}, C_{3}$ in the example, AESFilter will detect $Q_{5}$ as a matching simple subscription and $Q_{4}, Q_{3}$ as active complex subscriptions. Then YFilter $_{\sigma}$ is adapted to check only for the complex queries $Q_{4}^{\prime}$ and $Q_{3}^{\prime}$. Suppose that $Q_{4}^{\prime}$ only is verified. The matchings are $Q_{4}$ and $Q_{5}$.

$Y$ Filter $_{\sigma}$ is a modified version of the YFilter automaton. Given the set $\left\{Q_{i}^{\prime}\right\}$ of queries corresponding to the complex subscriptions, we construct a YFilter automaton. Now, given a tree $t$, only certain subscriptions are active so the automaton is virtually pruned to adapt to the specific filtering task for $t$. As shown in [8], this is a most efficient organization that scales with the number of subscriptions because it groups path queries based on their common linear prefixes.

Figure 5 describes the Filter's architecture consisting mainly of the three modules previously described. Dotted arrows represent the flow of data corresponding to the offline adjustment of the filter when the subscription database changes. Plain arrows correspond to the data flowing in the Filter during the processing of an XML document coming on the input stream. Input data for the Filter is figured in ovals.

Web service calls. To conclude this section, we consider a particular aspect of the filter, namely the use of external services. This is a place where the fact that the trees we monitor may be active (i.e., in ActiveXML) is particularly relevant. Such a tree may include calls to a Web service which provides, on demand, a part of the document that was considered too big to be passed into the stream. Let us consider the following XML document coming on a stream:

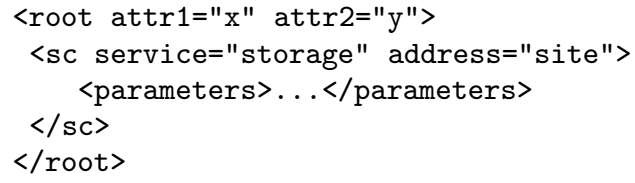

The active part is rooted at the element $s c$. Suppose that the call to service storage@site would evaluate to:

$$
\langle\mathrm{c}\rangle\langle\mathrm{d}\rangle \ldots\langle/ \mathrm{d}\rangle\langle/ \mathrm{c}\rangle
$$


This data would replace the subtree rooted at $s c$. Let us also assume that a subscription filters this stream with the query:

\$item.attr $1=" x "$ and $\$$ item.attr $2=" z "$ and $\$$ item $/ / c / d$

The Filter first evaluates the simple conditions, i.e. the conditions on the root attributes. Since the checking for the second condition fails, it will not pursue the checking of the XPath expression. Observe that by replacing the service call subtree with the result of the Web service call, the XPath expression would evaluate to true. Our strategy avoids the unnecessary call to service storage@site.

\section{STREAM REUSE}

This section presents P2PM's support for stream reuse.

$P 2 P M$ is a $\mathrm{P} 2 \mathrm{P}$ platform providing monitoring services. Services such as Selection, Join, Restructure or Publisher are provided by the peers but each peer does not have to support all services. For instance, a $P D A$ may refuse to support an expensive service such as Join whereas, an enterprise server may typically be willing to do it. When a new monitoring subscription is submitted to a Subscription Manager at a particular peer, an essential aspect of its work is to determine which already existing streams may be reused for that task to save CPU consumption and network traffic. Since monitoring subscriptions are tasks that execute over long periods of time, it is therefore important to pay once for optimizing the monitoring plan.

To support stream reuse, the system provides a Stream Definition Database that contains the description of all available streams. This database is implemented using the KadoP [3] system, a P2P XML index and repository over a DHT system. The motivation is that a centralized database would potentially be a bottleneck. All the peers in the KadoP network can participate in the storage and indexing of the Stream Definition Database. One can efficiently discover streams of interest even when millions of streams have been declared by tens of thousands of peers.

Stream representation. The description of streams is maintained in a database. The system provides services for publishing information about existing streams, and for querying this information in particular for stream reuse. The information about some stream is defined with XML data:

<Stream PeerId="..." StreamId="..." isAChannel="...">

$<$ Operator $>\ldots</$ Operator $>\langle$ Operands $>\ldots</$ Operands $>$

$<$ Stats $>\ldots</$ Stats $>$

$</$ Stream $>$

The pair (StreamId,PeerId) fully identifies the stream.

Operands provides the list of pairs (OPeerId,OStreamId) of operands, and Stats provides statistical information maintained for the stream such as the average volume of data in the stream for some period of time. The Operator argument specifies the operator that is used to produce this stream. When the set Operands is empty, this means that the stream is a monitoring source, in other words, it is produced by an alerter. The boolean attribute isAChannel specifies if the stream is published under the form of a channel or not. Recall that a channel is a stream that has been published. A channel is typically multicasted to several peers, so PeerId is not the single peer that can provide this data. The information about a particular channel is also defined in XML:

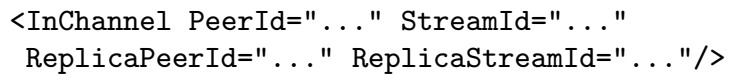

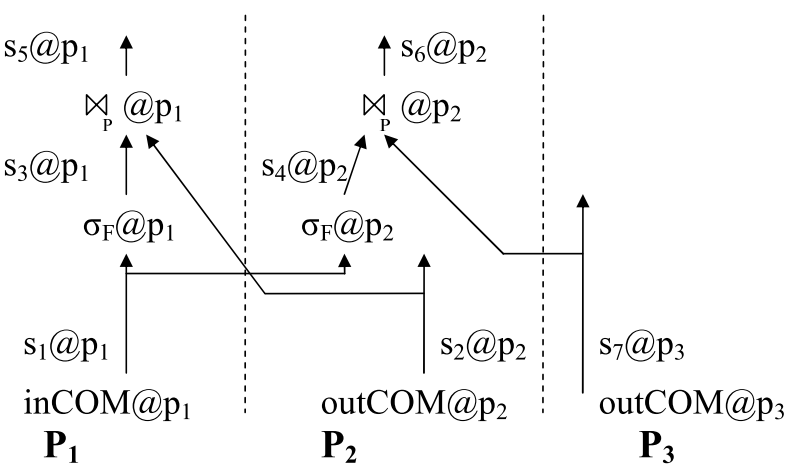

Figure 7: Stream Replication and Equivalence

Suppose peer $p$ published a stream $s$ in a channel and that peer $p^{\prime}$ subscribes to that channel. Then $p^{\prime}$ may choose to publish this information to let it be known that he can also provide $(p, s)$. To do so, it also has to provide an Id for the replica, $s^{\prime}$. The attributes would be in order $p, s, p^{\prime}$ and $s^{\prime}$.

When we publish the specification of a stream, we always do it with respect to the original streams and not to the replica. For instance, suppose that a peer provides some filtering of $s^{\prime} @ p^{\prime}$, say $s^{\prime \prime} @ p^{\prime \prime}$. When declaring this new stream, it will use $s @ p$ as operand. So, even if "physically" $s^{\prime \prime} @ p^{\prime \prime}$ is obtained by filtering $s^{\prime} @ p^{\prime}$, it is viewed semantically as a filtering of $s @ p$. This greatly facilitates the re-use of streams.

Algorithm for discovering useful streams. The algorithm searches for existing streams that can be used for serving a newly declared subscription. Suppose the subscription is a selection over the Web communications at peer $p_{1}$. One first queries the database to see if a communication alerter for $p_{1}$ exists. Say it does and its output stream is $s_{1} @ p_{1}$. Then one queries the database to see whether there is a filtering of $s_{1} @ p_{1}$ that performs the desired task (see Figure 7).

Since the operators have been published over the original streams, we are concerned only with searching operators on original versions of the streams. The issue of replicas comes only in a second stage, namely when we have discovered a stream we are interested in and when we have to select either this original stream or one of its replicas. This selection is guided by the optimizer. Typically, we select as provider a peer that is preferably "close" (networkwise) and not overloaded. Clearly, the notion of replica is not a full answer to stream equivalence. Indeed, one can find in Figure 7 an example of two streams that are equivalent (because of the equivalence of algebraic expressions) without being replicas. The Reuse algorithm works on a monitoring plan, trying to find sub-plans already supported by existing streams. Reuse starts its search from the sources of the monitoring stream. For instance, if a source stream in the subscription involves incoming communications at Peer $p_{1}$, we can use the following XPath query to find streams produced by alerters on $p_{1}$, assuming the variable $\$ p_{1}$ holds the peer ID:

/Stream [@PeerId = \$p1] [Operator/inCom]

Suppose now that we found that $s_{1}$ contains alerts on incoming calls and that we want some particular filter over $s_{1}$. The following query returns all possible candidates that filter $s_{1}$ assuming $\left(\$ p_{1}, \$ s_{1}\right)$ holds the peer and stream ID:

/Stream [Operator/Filter]

[Operands/Operand [@OPeerId=\$p1] [@OStreamId=\$s1] ]

Now consider the more complex monitoring plan corresponding to the evaluation of the following expression:

$$
\bowtie_{P}\left(\sigma_{F}\left(i n C O M @ p_{1}\right), \text { outCOM@ } p_{2}\right)
$$


Figure 7 shows existing streams in the system that may be used. Suppose that we already found that the filtering of incoming calls can be provided by $s_{3} @ p_{1}$ and $s_{4} @ p_{2}$; and that the outcalls can be provided by $s_{2} @ p_{2}$. To search for the join, we can ask the query:

/Stream
[Operator/Join]
[Operands/Operand [@OPeerId=\$p1] [@OStreamId=\$s3]]
[Operands/Operand [@OPeerId=\$p2] [@OStreamId=\$s2]

More generally, the algorithm proceeds from the "leaves" of the monitoring plan, attempting to map nodes in the plan to existing streams. Operators that have all their operands matched generate queries to the database. The result of the queries determines whether this operator will be mapped to an existing stream. For a node that is matched, the algorithm searches for possible replicas of the streams to substitute for that node. The nodes that have not been matched correspond to new streams that have to be produced.

\section{RELATED WORK}

Most of the works in the field of monitoring peer-to-peer systems have addressed two aspects. The first is the gathering of statistics for file sharing systems, e.g. [16], in order to answer queries such as: which is the most shared video file in this P2P system? The other is network monitoring typically for improving QoS, e.g. [14].

Our work differs greatly of these, since we are primarily interested in monitoring events regarding document updates (database, RSS feeds, Web pages) and distributed applications running in $\mathrm{P} 2 \mathrm{P}$ systems. For these reasons, this topic is at the confluence of two research areas : Web-scale monitoring systems and stream processing.

Systems such as [7], [12] and [15] do centralized monitoring for changes in documents on the Web. NiagaraCQ $[7]$ becomes scalable by regrouping similar structures of different continuous queries expressed in an XML-QL language. PeerCQ[10] is a P2P system that performs Web-scale information monitoring using continuous queries and implementing efficient algorithms for allocating the queries on peers. All the processing for a continuous query is done on a peer.

STREAM[13] processes data streams by transforming them into relations. The query results are transformed back into streams. This system uses time-based windows for bounding the necessary storage for the evaluation of joins over streams, for instance. We intend to couple this approach with an efficient garbage collection mechanism which detects and eliminates unnecessary trees from the storage. Borealis[1] and Aurora[2] are also stream processing engines. All these systems are based on the relational model, processing streams of data tuples.

A work close in spirit to ours is StreamGlobe[11, 17], a $\mathrm{P} 2 \mathrm{P}$ system for efficiently querying data streams represented in XML, that uses an XQuery-like language and proposes stream sharing to achieve scaling. StreamGlobe performs in-network search for useful streams while we are using a service (provided by a DHT) for maintaining and querying the stream definitions. Also StreamGlobe shares streams derived from data sources by applying only unary operators, e.g. selections, projections and window-aggregation while the system we present allows sharing for all streams. In particular, the stream resulting from the join of two streams can be shared, detected as useful and re-used.

\section{CONCLUSION}

In this paper we presented P2PM, a versatile peer-to-peer tool for monitoring generic $\mathrm{P} 2 \mathrm{P}$ systems. Alerters have to be developed for every type of system one wishes to monitor. However, the part of the architecture dedicated to processing and the stream publishers remain the same, regardless of the monitored application. We have shown an efficient filtering technique and an algorithm for detecting useful streams for covering (parts of) a new monitoring task.

We are currently testing our system by monitoring RSS feeds. We also plan to test our system for monitoring P2P systems running distributed applications like Edos[9], already mentioned. Certainly, we have met very interesting problems and we plan to explore them in the near future. One is defining and implementing an efficient garbage collection mechanism for reducing the storage needed for our stateful stream processors. A second is finding solutions for the issue of stream equivalence. We are also interested in detecting and reusing streams that hold sufficient data.

\section{REFERENCES}

[1] D. J. Abadi, Y. Ahmad, M. Balazinska, U. Çetintemel, M. Cherniack, J.-H. Hwang, W. Lindner, A. Maskey, A. Rasin, E. Ryvkina, N. Tatbul, Y. Xing, and S. B. Zdonik. The design of the Borealis stream processing engine. In $C I D R, 2005$.

[2] D. J. Abadi, D. Carney, U. Çetintemel, M. Cherniack, C. Convey, S. Lee, M. Stonebraker, N. Tatbul, and S. B. Zdonik. Aurora: a new model and architecture for data stream management. VLDB J., 12(2), 2003.

[3] S. Abiteboul, I. Manolescu, and N. Preda. Constructing and querying peer-to-peer warehouses of XML resources. In ICDE, 2005.

[4] S. Abiteboul, I. Manolescu, and E. Taropa. A framework for distributed XML data management. In EDBT, 2006.

[5] Active XML Survey, ftp://ftp.inria.fr/inria/projects/ gemo/gemo/gemoreport-331.pdf.

[6] Business Process Execution Language for Web Services, http://www.ibm.com/developerworks /library/ws-bpel/.

[7] J. Chen, D. J. DeWitt, F. Tian, and Y. Wang. NiagaraCQ: A scalable continuous query system for Internet Databases. In SIGMOD Conference, 2000.

[8] Y. Diao, P. M. Fischer, M. J. Franklin, and R. To. Yfilter: Efficient and scalable filtering of XML documents. In ICDE, 2002.

[9] Edos, http://www.edos-project.org.

[10] B. Gedik and L. Liu. PeerCQ: A decentralized and self-configuring Peer-to-Peer information monitoring system. In ICDCS, 2003.

[11] R. Kuntschke, B. Stegmaier, A. Kemper, and A. Reiser. Streamglobe: Processing and sharing Data Streams in Grid-Based P2P infrastructures. In VLDB, 2005.

[12] L. Liu, C. Pu, and W. Tang. WebCQ: Detecting and delivering information changes on the Web. In CIKM, 2000.

[13] R. Motwani, J. Widom, A. Arasu, B. Babcock, S. Babu, M. Datar, G. S. Manku, C. Olston, J. Rosenstein, and R. Varma. Query processing, approximation, and resource management in a data stream management system. In CIDR, 2003.

[14] Netscout, http://www.netscout.com/.

[15] B. Nguyen, S. Abiteboul, G. Cobena, and M. Preda. Monitoring XML data on the Web. In SIGMOD, 2001.

[16] Peermind, http://www.peermind.com/.

[17] B. Stegmaier, R. Kuntscke, and A. Kemper. Streamglobe: adaptive query processing and optimization in streaming P2P environments. In ACM International Conference Proceeding Series; Vol. 72, 2004.

[18] XQuery, http://www.w3.org/xml/query/. 